\title{
Asymptotic rejection of unknown sinusoidal disturbances in nonlinear systems *
}

\author{
Zhengtao Ding \\ Control Systems Centre, School of Electrical and Electronic Engineering, \\ University of Manchester, PO Box 88, Manchester M60 1QD, United Kingdom
}

\begin{abstract}
This paper deals with global disturbance rejection of nonlinear systems. The disturbance is assumed to be sinusoidal with completely unknown phases, amplitude, and frequencies, but the number of distinct frequencies or the order of the corresponding unknown linear exosystem is known. Different from the common structural assumptions of nonlinear systems needed in literature for disturbance rejection of nonlinear systems, the proposed method only requires the information of control design with a known Lyapunov function when the system is disturbance-free, and a mild assumption needed for internal model design. The proposed disturbance rejection algorithm extends complete global rejection of unknown sinusoidal disturbances for nonlinear dynamic systems beyond the common nonlinear models such as the strict feedback forms and the output feedback forms.
\end{abstract}

Key words: Disturbance rejection, Uncertainty, Nonlinear systems, Adaptive control

\section{Introduction}

Asymptotic rejection of sinusoidal disturbances has been intensively studied for linear systems (Feng and Palaniswami, 1991; Bodson, Sacks and Khosla, 1994; Bodson and Douglas, 1997; Marino, Santosuosso and Tomei, 2003), even when the disturbance frequencies are unknown (Bodson et al., 1994; Bodson and Douglas, 1997; Marino et al., 2003). For nonlinear systems, recent results

\footnotetext{
‡ This paper was not presented at any IFAC meeting. Tel. $+44-161-3064663$. Fax +44-161-3064647. This research was supported by EPSRC grant EP/C500156/1.

Email address: zhengtao.ding@manchester.ac.uk (Zhengtao Ding).
} 
for disturbance rejection of unknown sinusoidal disturbances are reported in (Nikiforov, 1998; Ding, 2003a; Ding, 2003b; Ding, 2006). The results obtained so far for nonlinear systems are restricted to the nonlinear systems in the output feedback form (Ding, 2003a; Ding, 2003b; Ding, 2006) via output feedback or in the strict feedback form via state feedback. A related problem is output regulation, of which the structural stable output regulation problem is solved for nonlinear systems with known exosystems in (Byrnes, Priscoli, Isidori and Kang, 1997).

In this paper, we deal with global rejection of unknown disturbances for nonlinear systems via state feedback, and extend the global rejection of unknown disturbances from systems in the strict feedback form and output feedback form to more general nonlinear systems. The conditions in the system structure are relaxed, and instead, an assumption is made on the existence of feedback control law of the system with a known Lyapunov function when the disturbance is zero. Since the exosystem is still unknown, adaptive techniques are used in dealing with the uncertainties in the combined control design. The proposed internal model depends on the final control input and system state variables. The proposed control design follows a parallel structure with additional term added to the control design for disturbance-free systems. Under the proposed control, the unknown disturbance is completely rejected. The proposed algorithm is demonstrated by an example with simulation results shown.

\section{Problem Formulation}

We consider a single-input nonlinear system

$$
\dot{x}=f(x)+g(x)(u-\mu)
$$

where $x \in R^{n}$ is the state vector, $u \in R$ is the control, $\mu$ is a disturbance, generated from an unknown exosystem

$$
\begin{aligned}
& \dot{w}=S w \\
& \mu=L^{T} w
\end{aligned}
$$

with $w \in R^{s}$

Assumption 1. The eigenvalues of $S$ are with zero real parts and are distinct, and $\left\{S, L^{T}\right\}$ is observable. 
Assumption 2. There exists a function $h(x): R^{n} \rightarrow R^{s}$ such that $\frac{\partial h(x)}{\partial x} g(x)=$ $G$, a nonzero constant vector in $R^{s}$.

Remark 1: This assumption is an condition for observability of the disturbances from the system state. If the vector field $g$ is a nonzero constant, there always exists a solution of $h(x)=H x$ for a nonzero $G$. For a general non-constant vector field $g(x)$, solutions can still be found. For the convenience of discussion, without loss of generality, we take $G=[0, \ldots, 0,1]^{T}$. Let $\Delta_{g}=\operatorname{span}\{g(x)\}$, and therefore $\Delta_{g}$ is involutive. From Frobenius theorem (Isidori, 1995), we can find $h_{i}(x), i=1, \ldots, s$, such that $L_{g} h_{i}(x)=0$ for $i=1, \ldots, s-1$, and $L_{g} h_{s}(x)=1$.

Assumption 3: For the disturbance-free system, there exists a state feedback control input $u=\alpha(x)$ such that the closed loop system is asymptotically stable. Furthermore, there exists a Lyapunov functions $V(x)$ such that

$$
\begin{array}{r}
\gamma_{1}(\|x\|) \leq V(x) \leq \gamma_{2}(\|x\|) \\
\frac{\partial V(x)}{\partial x}(f(x)+g(x) \alpha(x)) \leq-\gamma_{3}(\|x\|) \\
c_{1}\left|\frac{\partial V(x)}{\partial x} g(x)\right|^{c_{2}} \leq \gamma_{3}(\|x\|)
\end{array}
$$

where $\gamma_{i}, i=1,2,3$, are $K_{\infty}$ functions and $c_{i}, i=1,2$, are positive real constants with $c_{2}>1$.

Remark 2: The conditions specified in (3) and (4) are automatically satisfied if the closed-loop system is asymptotically stable. The condition (5) is always satisfied if the closed-loop system is exponentially stable. It is known that exponential stability can be achieved for a few classes of nonlinear systems, including the nonlinear systems in the strict feedback form. Note that there are systems such that the conditions in Assumption 3 are all satisfied, but the systems are not exponentially stable (Khalil, 2002).

The problem considered in this paper is to design a feedback control structure to asymptotically reject the unknown disturbance and to ensure the stability of the closed loop system.

\section{$3 \quad$ Internal Model Design}

For a nonzero $G$, there exists a Hurwitz matrix $F$ such that $\{F, G\}$ is controllable. In fact, there exists a nonsingular transform $T$ such that $G=T b$ with $b \in R^{s}$ and $b=[0, \ldots, 0,1]^{T}$. We can then choose $F=T A T^{-1}$ with $A$ being Hurwitz and 


$$
A=\left[\begin{array}{cccc}
-a_{1} & 1 & \ldots & 0 \\
\vdots & & \ddots & \\
-a_{s-1} & 0 & \ldots & 1 \\
-a_{s} & 0 & \ldots & 0
\end{array}\right]
$$

The internal model is designed as

$$
\dot{\xi}=F \xi-G u+F h(x)-\frac{\partial h(x)}{\partial x} f(x)
$$

It has a nice property as described in the following lemma.

Lemma 3.1 There exist a nonsingular matrix $M$, and positive real constants $d_{\epsilon}$ and $\lambda_{\epsilon}$ such that the biased error defined by

$$
\epsilon=M w-\xi-h(x)
$$

satisfies

$$
\|\epsilon(t)\| \leq d_{\epsilon} e^{-\lambda_{\epsilon} t}
$$

Proof: With $\{F, G\}$ controllable and $\left\{S, L^{T}\right\}$ observable and $F$ and $S$ having exclusively different spectra, there exists a unique nonsingular matrix $M$ such that

$$
M S-F M=-G L^{T}
$$

If we define $\eta=M w$, we have

$$
\begin{aligned}
& \dot{\eta}=F \eta-G q^{T} \eta \\
& \mu=q^{T} \eta
\end{aligned}
$$

where $q=M^{-T} L$. Then a directly evaluation gives

$$
\begin{aligned}
\dot{\epsilon}= & F \eta-G q^{T} \eta-F \xi+G u-F h(x)+\frac{\partial h(x)}{\partial x} f(x) \\
& -\frac{\partial h(x)}{\partial x}(f(x)+g(x)(u-\mu)) \\
= & F \epsilon
\end{aligned}
$$


It can be seen that $\epsilon$ is exponentially stable. In fact, define $V_{\epsilon}=\epsilon^{T} P \epsilon$ with $P$ and $Q$ being positive definite matrices satisfying $P F+F^{T} P=-Q$, and we have

$$
\frac{d V_{\epsilon}}{d t}=-\epsilon^{T} Q \epsilon \leq-\frac{\lambda_{\min }(Q)}{\lambda_{\max }(P)} V_{\epsilon}
$$

and therefore

$$
V_{\epsilon} \leq V_{\epsilon}(\epsilon(0)) e^{-\lambda_{\min }(Q) t / \lambda_{\max }(P)}
$$

and furthermore,

$$
\|\epsilon(t)\| \leq\|\epsilon(0)\| \sqrt{\frac{\lambda_{\max }(P)}{\lambda_{\min }(P)}} e^{-\frac{\lambda_{\min }(Q) t}{2 \lambda_{\max }(P)}}
$$

where $\lambda_{\min }(\cdot)$ and $\lambda_{\max }(\cdot)$ denote the minimum and the maximum eigenvalues of a matrix respectively.

Remark 3: The internal model (7) does not require the knowledge of $S$ and $L$.

\section{Control Design}

If the disturbance dynamics are known, based on the internal model (7), the control input can be designed as

$$
u=\alpha(x)+q^{T}(\xi+h(x))
$$

To deal with the unknown $q$, an adaptive control law is introduced as

$$
u=\alpha(x)+\hat{q}^{T}(\xi+h(x))
$$

where $\hat{q}$ is an estimate of $q$. The corresponding adaptive law is designed as

$$
\dot{\hat{q}}=-\Gamma \frac{\partial V(x)}{\partial x} g(x)(\xi+h(x))
$$

where $\Gamma$ is a positive definite matrix. 
Theorem 4.1 For a system (1) satisfying Assumptions 1 to 3, the control input (17) and the adaptive law (18) ensure the boundedness of all the variables and the asymptotically rejection of disturbances in the sense that $\lim _{t \rightarrow \infty} x(t)=0$.

Proof: Construct a dummy first order system

$$
\frac{d \bar{\epsilon}}{d t}=-\lambda_{\epsilon} \bar{\epsilon}, \bar{\epsilon}(0)=d_{\epsilon}
$$

where $d_{\epsilon}>0$ is the same positive constant as shown in (9), and hence $\bar{\epsilon}(t)>0$ for all $t \geq 0$. From Lemma 3.1, it follows that $\|\epsilon(t)\| \leq \bar{\epsilon}(t)$.

Define a Lyapunov function candidate

$$
W=V(x)+\frac{1}{2} \tilde{q}^{T} \Gamma^{-1} \tilde{q}+\frac{c_{3}}{c_{4}} \bar{\epsilon}^{c_{4}}
$$

where $\tilde{q}=q-\hat{q}, c_{3}$ and $c_{4}$ are positive real constants with $c_{4}=\frac{c_{2}}{c_{2}-1}$. Its derivative along the system dynamics (1) and the adaptive law (18) is given by

$$
\begin{aligned}
\dot{W}= & \frac{\partial V(x)}{\partial x}[f(x)+g(x)(u-\mu)]-\tilde{q}^{T} \Gamma^{-1} \dot{\hat{q}}-c_{3} \lambda_{\epsilon} \bar{\epsilon}^{c_{4}} \\
= & \frac{\partial V(x)}{\partial x}[f(x)+g(x) \alpha(x)]+\frac{\partial V(x)}{\partial x} g(x) \hat{q}^{T}(\xi+h(x))-\frac{\partial V(x)}{\partial x} g(x) q^{T} \eta \\
& +\tilde{q}^{T} \frac{\partial V(x)}{\partial x} g(x)(\xi+h(x))-c_{3} \lambda_{\epsilon} \bar{\epsilon}^{c_{4}} \\
\leq & -\gamma_{3}(\|x\|)+\frac{\partial V(x)}{\partial x} g(x)\left[\hat{q}^{T}(\xi+h(x))\right. \\
& \quad-q^{T}(\epsilon+\xi+h(x))+\tilde{q}^{T}(\xi+h(x)]-c_{3} \lambda_{\epsilon} \bar{\epsilon}^{c_{4}} \\
\leq & -\gamma_{3}(\|x\|)+\left|\frac{\partial V(x)}{\partial x} g(x) \| q^{T} \epsilon\right|-c_{3} \lambda_{\epsilon} \bar{\epsilon}^{c_{4}}
\end{aligned}
$$

Applying Young's inequality to the second term on the right hand side of (21) gives

$$
\left|\frac{\partial V(x)}{\partial x} g(x)\right|\left|q^{T} \epsilon\right| \leq \frac{c_{5}^{c_{2}}}{c_{2}}\left|\frac{\partial V(x)}{\partial x} g(x)\right|^{c_{2}}+\frac{1}{c_{4} c_{5}^{c_{4}}}\|q\|^{c_{4}}\|\epsilon\|^{c_{4}}
$$

where $c_{5}$ is any positive real constant. We set $c_{5}=\left(\frac{c_{1} c_{2}}{2}\right)^{1 / c_{2}}$ and $c_{3}=\frac{2}{c_{4} \lambda_{\epsilon}}\left(\frac{2\|q\|}{c_{1} c_{2}}\right)^{c_{4}}$, which results in

$$
\left|\frac{\partial V(x)}{\partial x} g(x)\right|\left|q^{T} \epsilon\right| \leq \frac{c_{1}}{2}\left|\frac{\partial V(x)}{\partial x} g(x)\right|^{c_{2}}+\frac{1}{2} \lambda_{\epsilon} c_{3} \bar{\epsilon}^{c_{4}}
$$


Substituting (23) into (21), we have

$$
\dot{W} \leq-\frac{1}{2} \gamma_{3}(\|x\|)-\frac{1}{2} c_{3} \lambda_{\epsilon} \bar{\epsilon}^{c_{4}}
$$

Therefore we conclude that all the variables are bounded. Furthermore, from the invariant set theorem, we have $\lim _{t \rightarrow \infty} x(t)=0$.

\section{An Example}

Consider a nonlinear system

$$
\begin{aligned}
& \dot{x}_{1}=2 x_{2}+x_{1}^{2}+u-\mu \\
& \dot{x}_{2}=-x_{2}+\frac{1}{1+x_{2}^{2}}(u-\mu)
\end{aligned}
$$

where the disturbance $\mu$ is an unknown sinusoidal signal generated by the exosystem with

$$
S=\left[\begin{array}{cc}
0 & -\omega \\
\omega & 0
\end{array}\right]
$$

Remark 4: There are a number of physical models with constant $g(x)$ vectors in (Marino and Tomei, 1995; Krstic, Kanellakopoulos and Kokotovic, 1995) to which the proposed disturbance rejection method can be applied. Here, we choose an example with a non-constant vector field $g(x)=\left[1, \frac{1}{1+x_{2}^{2}}\right]^{T}$ to demonstrate the proposed method.

The disturbance-free system is stabilized by setting $u=\alpha(x)$ with

$$
\alpha(x)=-6 x_{1}+x_{2}+x_{1} x_{2}-2 x_{1}^{2}+x_{2}^{2}+\frac{1}{2} x_{1} x_{2}^{3}
$$

In fact, if we choose

$$
V(x)=\frac{1}{2} x_{1}^{2}+\frac{1}{2}\left(x_{2}+\frac{1}{3} x_{2}^{3}-x_{1}\right)^{2}
$$

we have 


$$
\begin{aligned}
\frac{\partial V(x)}{\partial x}(f(x)+g(x) \alpha(x)) & =-3 x_{1}^{2}-3\left(x_{2}+\frac{1}{3} x_{2}^{3}-x_{1}\right)^{2} \\
\frac{\partial V(x)}{\partial x} g(x) & =x_{1}
\end{aligned}
$$

With reference to Assumption 3, we have

$$
\begin{array}{r}
\frac{3+\sqrt{5}}{4}\|x\|^{2} \leq V(x) \leq\left(2+\left(1+\frac{1}{3}\|x\|^{2}\right)^{2}\right)\|x\|^{2} \\
\frac{\partial V(x)}{\partial x}(f(x)+g(x) \alpha(x)) \leq-\frac{9+3 \sqrt{5}}{2}\|x\|^{2} \\
\frac{9+3 \sqrt{5}}{2}\left|\frac{\partial V(x)}{\partial x} g(x)\right|^{2} \leq \frac{9+3 \sqrt{5}}{2}\|x\|^{2}
\end{array}
$$

Therefore the system with the disturbance-free control design satisfies Assumption 3 with $\gamma_{1}(\mu)=\frac{3+\sqrt{5}}{4} \mu^{2}, \gamma_{2}(\mu)=\left(2+\left(1+\frac{1}{3} \mu^{2}\right)^{2}\right) \mu^{2}, \gamma_{3}(\mu)=\frac{9+3 \sqrt{5}}{2} \mu^{2}$, and $c_{1}=\frac{9+3 \sqrt{5}}{2}, c_{2}=2$. With

$$
h(x)=\left[\begin{array}{ll}
x_{1} & x_{2}+\frac{1}{3} x_{2}^{3}
\end{array}\right]^{T}
$$

we have $G=\frac{\partial h(x)}{\partial x} g(x)=\left[\begin{array}{ll}1 & 1\end{array}\right]^{T}$. Hence, Assumption 2 is also satisfied. Note that Assumption 1 is automatically satisfied from the statement that disturbance is a sinusoidal function. Based on the proposed control, the internal model, the adaptive law and the control input are designed as

$$
\begin{aligned}
& \dot{\xi}=F \xi-G u+\left[\begin{array}{c}
-4 x_{1}-x_{2}-x_{1}^{2}+\frac{1}{3} x_{2}^{3} \\
-6 x_{1}+2 x_{2}+\frac{4}{3} x_{2}^{3}
\end{array}\right] \\
& \dot{\hat{q}}=-x_{1} \Gamma\left[\begin{array}{c}
\xi_{1}+x_{1} \\
\xi_{2}+x_{2}+\frac{1}{3} x_{2}^{3}
\end{array}\right] \\
& u=\alpha(x)+\hat{q}^{T}\left[\begin{array}{c}
\xi_{1}+x_{1} \\
\xi_{2}+x_{2}+\frac{1}{3} x_{2}^{3}
\end{array}\right]
\end{aligned}
$$

Simulation studies have been carried out with

$$
F=\left[\begin{array}{ll}
-4 & 1 \\
-6 & 1
\end{array}\right], \quad \Gamma=10 I
$$




$$
\mu(t)=\left\{\begin{array}{cc}
\sin 2 t & \text { for } 0 \leq t<25 \\
\sin t \quad \text { for } 25 \leq t
\end{array}\right.
$$

The state variables and the control input are shown in Figure 1, and the estimated disturbance $\hat{q}^{T}(\xi+h(x))$ are shown in Figure 2 .
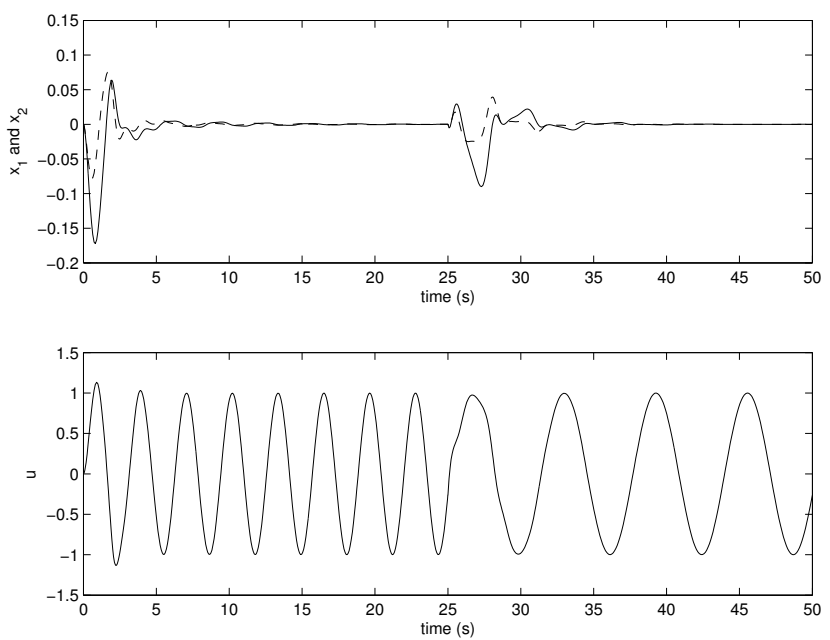

Fig. 1. State variable $x$ and input $u$.

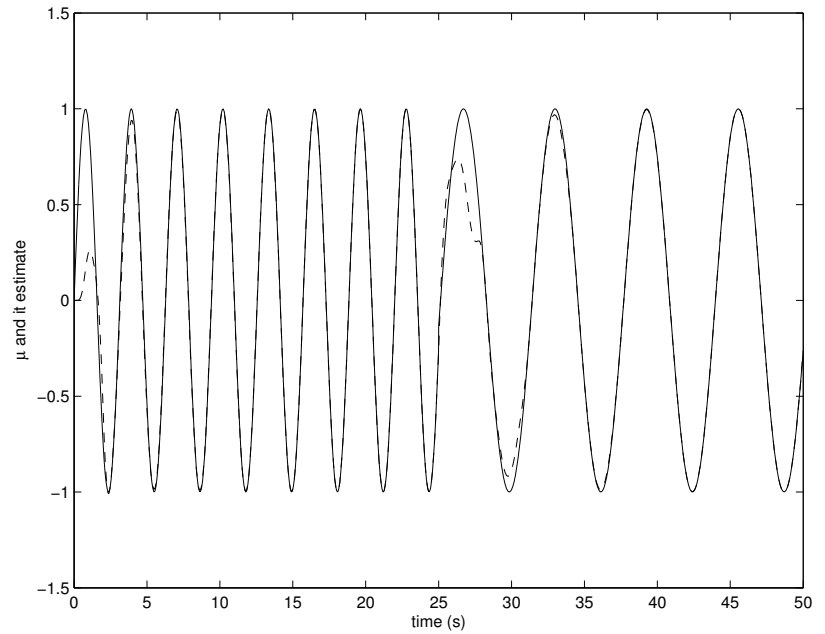

Fig. 2. Disturbance $\mu$ and its estimate.

\section{Conclusions}

A disturbance rejection algorithm is proposed for complete rejection of unknown sinusoidal disturbances for nonlinear systems whose control design for disturbance-free case is available. The dynamics of the proposed internal model 
depends on state and the control input. Adaptive control techniques are then used in designing the control input which consists of the control input for the disturbance-free system and an estimate of the unknown disturbance based on the internal model and an adaptive output gain. The proposed disturbance rejection design makes it possible to globally completely reject unknown sinusoidal disturbances for general nonlinear systems which may not be in the strict feedback form nor in the output feedback form.

\section{References}

Bodson, M. and Douglas, S. C. (1997). Adaptive algorithms for the rejection of sinusoidal disturbances with unknown frequencies. Automatica, 33(10), 22132221.

Bodson, M., Sacks, A. and Khosla, P. (1994). Harmonic generation in adaptive feedforward cancellation schemes. IEEE Trans. Automat. Contr., 39(9), 19391944.

Byrnes, C. I., Priscoli, F. D., Isidori, A. and Kang, W. (1997). Structural stable output regulation of nonlinear systems. Automatica, 33(3), 369-382.

Ding, Z. (2003a). Global stabilization and disturbance suppression of a class of nonlinear systems with uncertain internal model. Automatica, 39(3), 471-479.

Ding, Z. (2003b). Universal disturbance rejection for nonlinear systems in output feedback form. IEEE Trans. Automatic Control, 48(7), 1222-1226.

Ding, Z. (2006). Adaptive estimation and rejection of unknown sinusoidal disturbances in a class of non-minimum-phase nonlinear systems. IEE Proc.Control Theory Appl., 152(4), 379-386.

Feng, G. and Palaniswami, M. (1991). Unified treatment of internal model principle based adaptive control algorithms. International Journal of Control, 54, 883901.

Isidori, A. (1995). Nonlinear Control Systems. 3rd edn. Springer-Verlag. Berlin.

Khalil, H. K. (2002). Nonlinear Systems. 3rd edn. Prentice Hall. New Jersey.

Krstic, M., Kanellakopoulos, I. and Kokotovic, P. V. (1995). Nonlinear and Adaptive Control Design. John Wiley \& Sons. New York.

Marino, R., Santosuosso, G. L. and Tomei, P. (2003). Robust adaptive compensation of biased sinusoidal disturbances with unknown frequencies. Automatica, 39, 1755-1761.

Marino, R. and Tomei, P. (1995). Nonlinear Control Design: Geometric, Adaptive, and Robust. Prentice-Hall. London.

Nikiforov, V. O. (1998). Adaptive non-linear tracking with complete compensation of unknown disturbances. European Journal of Control, 4, 132-139. 